\title{
On Machine Learning Trends for Android Applications
}

\author{
Nived J. Baria \\ Department of Computer \\ Engineering \\ SVM Institute of Technology \\ Bharuch 392-001, Gujarat, India
}

\author{
Pruthvirajsinh S. Solanki \\ Department of Computer \\ Engineering \\ SVM Institute of Technology \\ Bharuch 392-001, Gujarat, India
}

\author{
Dhaval B. Rank \\ Department of Computer \\ Engineering \\ SVM Institute of technology \\ Bharuch 392-001, Gujarat, India
}

\author{
Tejasvi G. Kathiriya \\ Department of Computer Engineering \\ SVM Institute of Technology \\ Bharuch 392-001, Gujarat, India
}

\author{
Rutvij H. Jhaveri \\ Department of Computer Engineering \\ SVM Institute of Technology \\ Bharuch 392-001, Gujarat, India
}

\begin{abstract}
In recent past, machine learning has become the backbone of distinct mobile applications. In this paper, first we will discuss how machine learning is introduced as a pervasive field in the digital world. We carried out Google trends analysis of machine learning by taking various aspects into consideration. Moreover, we discovered the popularity of Android applications over their peer competitors. Additionally, we have provided an insight for a range of popular Android applications which uses machine learning techniques and a zoo of features which have been addressed using machine learning. The paper emphasizes the importance of machine learning in the development of different trending Android applications. Furthermore, it categorizes different applications by considering various available metrics in Google Play.
\end{abstract}

\section{Keywords}

Machine Learning, Android Application, Trend, artificial Intelligence.

\section{INTRODUCTION}

In the last few years, there has been upsurge of scrutiny for machine learning. Machine learning is a technique by which a computer continuously improves its efficiency to perform certain operations by using some algorithms which include analysis of past results and data [1]. The computer system learns from the historical results and acts according to that [2].

Artificial intelligence is an approach for making machines to think with the help of distinct reckoning methods [3]. The difference between machine learning

And artificial intelligence is that machine learning represents the machine which is learning something based on the past results data and artificial intelligence represents the approach for machine to think itself. Saying that, machine learning and artificial intelligence are often used interchangeably.

Machine learning is solving many issues of the digital world such as automated decision making, recommendations, anomaly detection, medical diagnosis and many more. Apart from this, machine learning is also used for high-speed corner detection [4], cyber security [5] and sentiment classification [6].There are three categories of machine learning algorithms: (1) Supervised learning (2) Unsupervised learning and (3) Reinforcement learning. In supervised learning, according to that data inputs, the supervised algorithm generates a functional map to obtain the desired output [7]. In unsupervised learning there is no resultant attribute to estimate. One of the examples of unsupervised learning is customer segmentation. Reinforcement learning is based on maximizing rewards in which a machine learns what things to do and how to act in specific situations. Machine learning algorithms help machine to minimize the errors and reduce complexity by learning from the historical data [8], [9]. Nowadays machine learning is flourishing in every mobile platform, especially in Android systems. Many of the popular android applications are based on distinct machine learning algorithms.

In this paper, first we compare Google trends results of 'machine learning' and 'artificial intelligence' by taking distinct parameters. Moreover, we carry out detailed review of various popular Android applications of distinct categories.

The paper is organized as follows. Section 2 graphically shows and discusses the current trends in machine learning. Section 3 reviews distinct Android applications in detail. Finally, Section 4 concludes the paper.

\section{TREND ANALYSIS OF MACHINE LEARNING}

Selection of a specific field of study is a critical task and therefore, one must analyse the current inclination of the field. Studying a currently trending field or a field with a future potential may prove to be more beneficial. In this section, we use Google trends to compare searching trends in machine learning as well as in artificial intelligence. All graphs in this section are based on data collected from Google trends.

\subsection{Web search trend analysis}

Figure 1 shows the Google trend for web search of machine learning and artificial intelligence for the year 2017. It depicts that both the topics are trending in the year and have great potential to rise in the upcoming year 2018 .

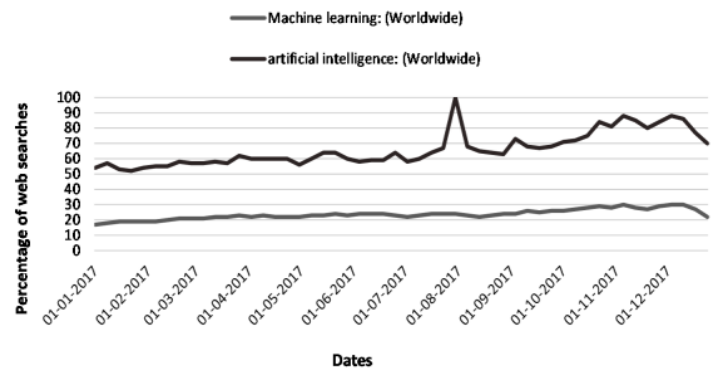

Figure 1.Google trends web search analysis for 2017 
Figure2 shows the Google trend for web search of machine learning and artificial intelligence for past 5 years of web search history. The rising graph shows that the fields are booming in recent years. Moreover, 2016 is the breakeven year for both the fields. According to the article published by Forbes magazine in early 2016 machine learning has been a main point of focus and interest for most of the majors of software industry. Many companies such as Google and Facebook made some of their resources open source for the people who wanted to carry out research in the field of machine learning. With this, many of the developers and freelancers got the resources they needed to carry on their work and this way machine learning became a trending field.

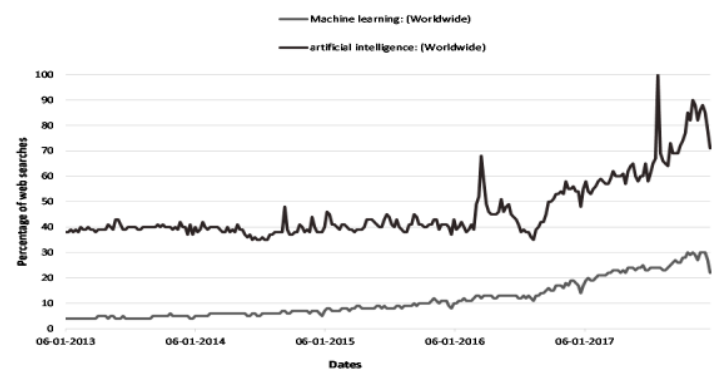

Figure 2. Google trends web search analysis for 2012-2017

\subsection{News search trend analysis}

Figure3shows the Google trend for news search of machine learning and artificial intelligence for the year 2017. Both the fields have caught eyes of media in the year 2017.

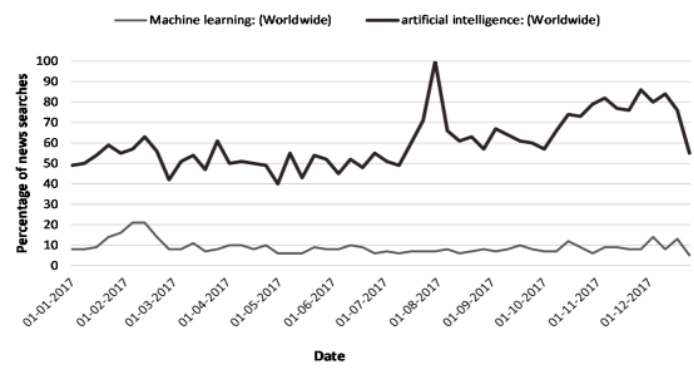

Figure 3.Google trends news search analysis for 2017

Figure4shows the Google trend for news search of machine learning and artificial intelligence for past 5 years. The graph again proves that the year 2016 has been an important year for both the fields.

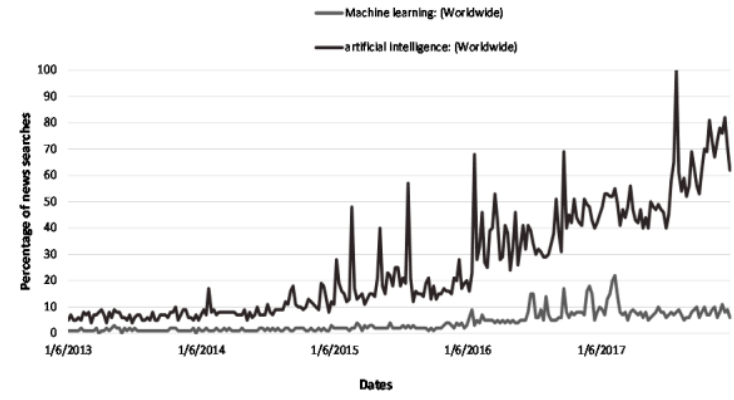

Figure 4.Google trends news search analysis for 2012-2017

The aforementioned graphs and analysis show that machine learning is a trending field and has a great future potential.

\section{REVIEW OF POPULAR ANDROID APPLICATIONS}

As per the analysis which we have done on the google trends we have seen that android is more trending than iOS,due to this reason we select android applications for the analysis. The following table depicts the category, user ratings and downloads (as per the Google Play till $31^{\text {st }}$ December, 2017) of some of the popular Android applications which employ meachine learning to implement various features. All the reviewed applications are given 4.0 or above ratings out of 5 .

Table 1. List of Android Apps using Machine Learning

\begin{tabular}{|c|c|c|c|}
\hline $\begin{array}{l}\text { APP } \\
\text { NAME }\end{array}$ & Category & Ratings & $\begin{array}{l}\text { Downloa } \\
\text { ds }\end{array}$ \\
\hline ESEA & Game & 4.6 & 1 Million \\
\hline $\begin{array}{ll}\text { Left } & \text { Vs } \\
\text { Right } & \end{array}$ & Game & 4.5 & 1 Million \\
\hline Carat & Supporting tool & 4.0 & $100 \mathrm{~K}$ \\
\hline Snap chat & Social media & 4.1 & $\begin{array}{l}500 \\
\text { Million }\end{array}$ \\
\hline Instagram & Social media & 4.5 & 1 Billion \\
\hline YouTube & Social media & 4.3 & 1 Billion \\
\hline Google & Supporting tool & 4.4 & 1 Billion \\
\hline $\begin{array}{l}\text { Aipoly } \\
\text { vision }\end{array}$ & Supporting tool & 4.2 & $10 \mathrm{~K}$ \\
\hline $\begin{array}{l}\text { SwiftKey } \\
\text { Neural }\end{array}$ & Supporting tool & 4.5 & $\begin{array}{l}100 \\
\text { Million }\end{array}$ \\
\hline $\begin{array}{l}\text { Sea Hero } \\
\text { Quest }\end{array}$ & Game & 4.8 & 1 Million \\
\hline $\begin{array}{l}\text { Train Your } \\
\text { Tic Tac Toe }\end{array}$ & Game & 4.3 & 100 \\
\hline WhatsApp & Social media & 4.4 & 1 Billion \\
\hline $\begin{array}{l}\text { Skillz- } \\
\text { Logical } \\
\text { Brain }\end{array}$ & Game & 4.7 & 5 Million \\
\hline B612 & Supporting tool & 4.4 & $\begin{array}{l}100 \\
\text { Million }\end{array}$ \\
\hline LeafSnap & Supporting tool & 4.0 & 972 \\
\hline Dango & Game & 4.4 & $50 \mathrm{k}$ \\
\hline $\begin{array}{l}\text { ImprompD } \\
\text { o }\end{array}$ & Supporting tool & 5.0 & 100 \\
\hline NewsC & Social media & 4.5 & $1 \mathrm{~K}$ \\
\hline SEER & Supporting tool & 4.3 & $5 \mathrm{~K}$ \\
\hline $\begin{array}{l}\text { ES File } \\
\text { explorer }\end{array}$ & Supporting tool & 4.6 & 4 million \\
\hline Tinder & Social media & 4.0 & 2 million \\
\hline Happen & Social media & 4.4 & $855 \mathrm{~K}$ \\
\hline Netflix & Social media & 4.4 & $\begin{array}{l}100 \\
\text { million }\end{array}$ \\
\hline Google & Supporting tool & 4.4 & 100 \\
\hline
\end{tabular}




\begin{tabular}{|l|l|l|l|}
\hline translate & & & million \\
\hline iTranslate & Supporting tool & 4.4 & 5 million \\
\hline Skillz & Game & 4.7 & 5 million \\
\hline Gboard & Supporting tool & 4.2 & 5 Billion \\
\hline Facebook & Social media & 4.0 & 1 Billion \\
\hline Pinterest & Social media & 4.6 & $\begin{array}{l}100 \\
\text { Million }\end{array}$ \\
\hline
\end{tabular}

Most of the games use machine learning algorithms to analyse the players gaming data and operate according to that after recommending difficulty level high or low. Additionally, for multiplayer games machine learning is employed to find a server on which other players are as skilled as the user.

Social media applications employ machine learning to provide suggestions about friends or invites, notifications about the topics of user interest or quicker answers to the text messages by learning words from the text.

Moreover, there are applications for nearly every task provided by the operating system in a mobile device. However, for additional functionalities users download external applications to perform various tasks in a better way. These supporting tools provide distinct features using machine learning algorithms.

Figure 5 shows percentage-wise division of reviewed applications according to the aforementioned three categories.

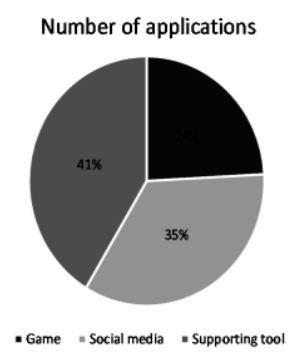

Figure5. Percentage-wise division of reviewed applications

Figure6shows a graph based on the average number of downloads of the applications of each categories. The data is obtained by simply calculating an average of the total downloads of all the applications falling into a particular category. The graph suggests that games are of less interest of whereas social media applications and supporting tools are of greater interests.

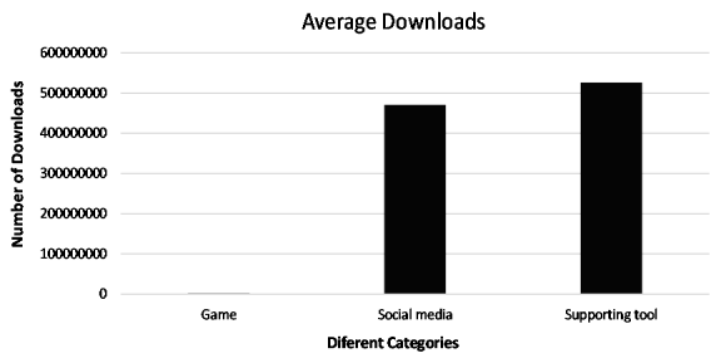

Figure6.Comparison of category-wise average downloads
Figure 7 shows the average rating of applications from each of the categories. The graph depicts that gaming applications received higher ratings whereas social media applications and supporting tools need more attention from developers in order to provide improved performance by efficient use of machine learning techniques.

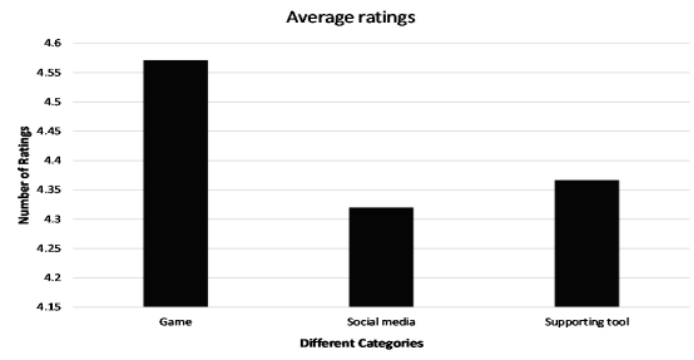

Figure 7.Comparison of category-wise average ratings

\section{CONCLUSION}

In this paper, we discover the potential of the machine learning field by using Google trends. With the ever increasing amount of data and significant rise in the use of smart devices, data analysis with machine learning would become integrated and imperative component in technological progress. The purpose of this paper is to provide the reader with an overview of distinct popular mobile applications which employ machine learning techniques. These applications use machine learning to provide recommendations, detailed analysis, predictions, and security along with categorization. However, still a considerable amount of research needs to be done for efficient use of machine learning techniques for distinct mobile applications. We can conclude that machine learning is the area which has a great potential in the field of mobile applications.

\section{REFERENCES}

[1] Y. Ba, "miRNomics: MicroRNA Biology and Computational Analysis," pp. 105-128, 2014.

[2] R. Society, Machine learning: the power and promise of computers that learn by example, 2017, p. 125.

[3] S. J. Russell, A Modern Approach, 1995, pp. 106-10.

[4] E. Rosten, "Machine Learning for High Speed Corner Detection," Computer Vision -- ECCV 2006, pp. 430443, 2004.

[5] P. K. Chan, "Machine learning for computer security," The Journal of Machine Learning Research, vol. 7, pp. 2669-2672, 2006.

[6] B. Pang, "Thumbs up? Sentiment Classification using Machine Learning Techniques," Proceedings of the ACL-02 Conference on Empirical Methods in Natural Language Processing - EMNLP '02, vol. 10, no. July, pp. 79-86, 2001.

[7] Y. k. Anish Talwar, "Machine Learning: An artificial intelligence methodology," International Journal of Engineering and Computer Science, vol. 2, no. 12, pp. 3400-3405, 2013.

[8] P. H. G. Brett Wujek, "Best Practices for Machine Learning Applications," pp. 1-23, 2016.

[9] P. Domingos, "A few useful things to know about machine learning," Communications of the ACM, vol. 10, no. 78, p. 55, 2012. 